\title{
Chasing behavior and optomotor following in free-flying male blowflies: flight performance and interactions of the underlying control systems
}

\author{
Christine Trischler, Roland Kern and Martin Egelhaaf* \\ Neurobiology and Center of Excellence, Cognitive Interaction Technology, Bielefeld University, Bielefeld, Germany
}

Edited by:

Martin Giurfa, Centre National de la Recherche Scientifi que - Université Paul Sabatier-Toulouse III, France

\section{Reviewed by:}

John Layne, University of Cincinnati, USA

Stephane Viollet, University of the Mediterranean Aix-Marseille II, France

*Correspondence.

Martin Egelhaaf, Neurobiology and Center of Excellence, Cognitive Interaction Technology, Bielefeld University, D-33501 Bielefeld,

Germany.

e-mail: martin.egelhaaf@uni-bielefeld.de
The chasing behavior of male blowflies after small targets belongs to the most rapid and virtuosic visually guided behaviors found in nature. Since in a structured environment any turn towards a target inevitably leads to a displacement of the entire retinal image in the opposite direction, it might evoke optomotor following responses counteracting the turn. To analyze potential interactions between the control systems underlying chasing behavior and optomotor following, respectively, we performed behavioral experiments on male blowflies and examined the characteristics of the two flight control systems in isolation and in combination. Three findings are particularly striking. (i) The characteristic saccadic flight and gaze style - a distinctive feature of blowfly cruising flights - is largely abandoned when the entire visual surroundings move around the fly; in this case flies tend to follow the moving pattern in a relatively continuous and smooth way. (ii) When male flies engage in following a small target, they also employ a smooth pursuit strategy. (iii) Although blowflies are reluctant to fly at high background velocities, the performance and dynamical characteristics of the chasing system are not much affected when the background moves in either the same or in the opposite direction as the target. Hence, the optomotor following response is largely suppressed by the chasing system and does not much impair chasing performance.

Keywords: visual pursuit, optomotor following, saccade, eye movements, fly, control systems

\section{INTRODUCTION}

Visual pursuit of small objects is an important task to be solved by many animals including humans, for instance when catching a baseball (e.g. McBeath et al., 1995; Land and McLeod, 2000; Shaffer and McBeath, 2002). Especially with respect to rapidity and virtuosity also various insect species show an impressive performance in pursuit tasks, for instance in the context of predation behavior (dragonflies: O'Carroll, 1993; Olberg et al., 2000, 2005; praying mantis: Rossel, 1980) or mating behavior (various fly species: Land and Collett, 1974; Collett and Land, 1975; Collett, 1980; Wagner, 1986b; Land, 1993a,b; Boeddeker et al., 2003).

Visual pursuit requires the target to be detected, then to be fixated in the frontal visual field and eventually to be followed by appropriate movements of the eyes, the head and/or the entire body. In primates visual pursuit is generally assumed to be based on a smooth control system mediating continuous rotations of the eyes, sometimes in combination with head and body movements (Miles, 1997; Schweigart et al., 1997; Krauzlis, 2004). However, pursuit responses are not always smooth. If target velocity is too fast, a rapid saccadic eye movement shifts the image of the object of interest from an eccentric retinal location to the fovea before smooth pursuit commences again. Smooth pursuit is not a distinguishing ability of primates, since male blowflies as well as other insects employ very precise smooth head and body movements to keep the image of a moving target fixated in the frontal visual field even during highly aerobatic flight maneuvers. During pursuit the angular velocity of the male blowfly was concluded to be controlled by the target's retinal position relative to the head's frontal midline ('error angle') (Boeddeker and Egelhaaf, 2003; Boeddeker et al., 2003).

Pursuit is complicated by the fact that any fixation turn towards a target in a structured environment inevitably leads to a displacement of the entire retinal image of the environment in the opposite direction of the turn. This wide-field motion may then evoke an optomotor following response that drives the animal to turn away from the moving target and, as a consequence, impedes pursuit. Following responses to wide-field motion of the eyes, the head and/or the entire body, though different in detail, have been found in a wide range of animals, from insects to primates including humans (reviews in Miles and Wallman, 1993); for insects see, e.g. Götz, 1968; Collett, 1980; Heisenberg and Wolf, 1984; Warzecha and Egelhaaf, 1996; Mronz and Lehmann, 2008). Thus, the two visually driven following behaviors, pursuit of small objects and following responses to wide-field motion may be in conflict with each other. This problem does not only arise during visual pursuit, but, for instance, also if a moving sound source is to be tracked in a visually structured environment during phonotactic behavior of certain insect species (e.g. Stout et al., 1987; Weber et al., 1987; Böhm et al., 1991).

Various schemes have been proposed of how optomotor following may interact with target pursuit in biological systems (e.g. Crapse and Sommer, 2008). Three such interaction schemes have been most widely discussed and modeled (e.g. Virsik and Reichardt, 1976; Collett, 1980; Webb et al., 2004). It is common to all these 
schemes that the turning commands mediated by the pursuit system and the optomotor system, respectively, are added to the overall turning response of the animal. However, the interaction schemes differ considerably with respect to the way they cope with the interference of optomotor following with pursuit (Figure 1).

(1) Additive scheme (Figure 1A): This scheme is the most parsimonious one, as the outputs of the two pathways are just added and do not interact in any other way. As a consequence,

\section{A Additive scheme}

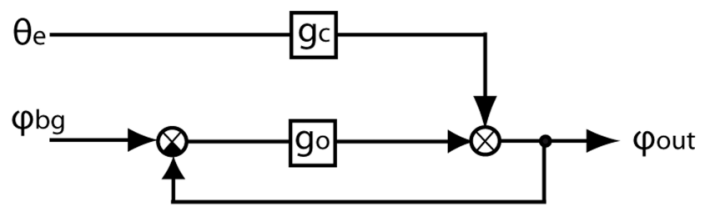

B Efference copy scheme



C Suppressive scheme

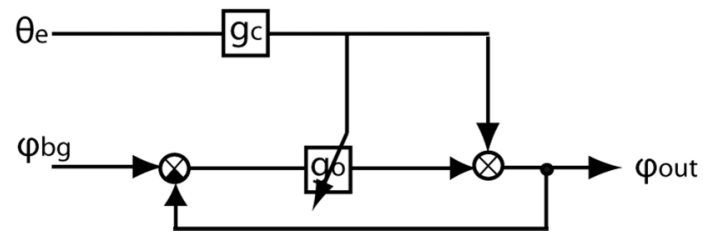

FIGURE 1 | Flow diagrams of three possible forms of interaction between the chasing system and the optomotor following system. The input to the chasing system is $\theta_{e^{\prime}}$ i.e. the error angle of the target relative to the frontal midline of the fly's visual field. $g_{c}$ and $g_{o}$ are the internal gains of the chasing system and the optomotor system, respectively. The input to the optomotor system is provided by $\varphi_{\mathrm{bg}}$, i.e. the angular velocity of the environment (i.e. background). $\varphi_{\text {out }}$ is the final turning response of the animal which is the sum of the turning responses mediated by the optomotor system and the chasing system. (A) Additive scheme: The signals mediating the turning responses of the chasing and the optomotor system are just added. There are no other interactions within the nervous system between the two system. (B) Efference copy scheme: As the additive scheme; however a copy of the turning command ('efference copy') is added to the input signal of the optomotor system to cancel during chasing behavior the visual feedback resulting from turns evoked by the optomotor system. (C) Suppressive scheme: A copy of the chasing control signal does not interact with the optomotor input signal in a linear way, as does the efference copy scheme, but reduces the internal optomotor gain or even suppresses the optomotor signal completely. Gain control has the advantage that the copy of the chasing signal does not need to be exactly adjusted to the visual feedback which cannot easily be predicted under natural flight conditions (A,B modified from Collett, 1980). the angular velocity of the pursuer depends on both the error angle and the wide-field motion caused by target pursuit in a stationary environment. The interference of the optomotor system is the smaller the larger the gain of the pursuit system relative to the optomotor gain (Virsik and Reichardt, 1976; Collett, 1980). It has been proposed that pursuit efficiency may be enhanced if the two interacting systems differ with respect to their dynamical properties, the pursuit system being mainly active at high and the optomotor system mainly at low frequencies (Collett, 1980; Egelhaaf, 1987, 1989).

(2) Efference copy scheme (Figure 1B): A so-called 'efference copy' is generated by every turning command of the pursuit system and thought to cancel out by subtraction the responses of the optomotor system to the visual consequences of target pursuit (von Holst and Mittelstaedt, 1950; Collett, 1980; Webb et al., 2004). Still, the pursuing fly's angular velocity depends on both the error angle and wide-field motion (Collett, 1980).

(3) Suppressive scheme (Figure 1C): In its most extreme version this scheme assumes that optomotor following is simply inoperative during turns evoked during pursuit; however, not as consequence of the limited dynamic range of the optomotor system, but as a result of active inhibition mediated by the pursuit system. Hence, any optomotor information is largely suppressed during pursuit (Webb et al., 2004). This disadvantage might be balanced by the advantage of simplicity in that the size of the expected optomotor signal does not have to be perfectly predicted as is the case for the efference copy scheme (Webb et al., 2004).

Although the wiring principles underlying these different interaction schemes differ considerably, it is not easy to distinguish between them experimentally when analyzing behavioral performance in stationary environments (Collett, 1980; Webb et al. 2004). However, if the environment is artificially moved around the animal in an experimental setup, the suppressive scheme, at least in its perfect form, may be distinguished from both the additive and the efference copy scheme. For the latter schemes the angular velocity of the animal and, thus, its pursuit performance should depend on the angular velocity of the background in contrast to the suppressive scheme.

Indeed, pursuit performance has been found to be affected by optomotor following in a number of studies on both primates and insects The steady-state pursuit eye velocity of primates induced by a small moving target was found to increase when the background moved in the same direction as the pursued target, and decreased when the background moved in the opposite direction as the target (Masson et al. 1995). Other behavioral studies indicate that pursuit eye movements are variously affected by a stationary or dynamic visual background (Collewijn and Tamminga, 1984; Keller and Khan, 1986; Kimmig et al., 1992; Masson et al., 1995; Mohrmann and Thier, 1995; Niemann and Hoffmann, 1997; Spering and Gegenfurtner, 2007). Despite discrepancies in detail, these studies indicate that the control systems mediating wide-field following and small-field pursuit in primates do not work independently of each other, at least at the behavioral level. In insects the possible impact of optomotor following on the performance of target pursuit has been addressed, so far, in only 
few studies. In praying mantis target pursuit is strongly affected by retinal background motion (Rossel, 1980). Similarly, in freeflying hoverfly Syritta or in tethered flying female houseflies target fixation and pursuit behavior are influenced by a simultaneously presented large-field background motion (Virsik and Reichardt, 1976; Collett, 1980).

Here we show by systematic behavioral analysis that male blowflies chasing after moving targets behave differently in this respect than most other animals analyzed so far. Although free-flying blowflies respond strongly with following responses when exposed to external wide-field motion, the same wide-field motion does not much deteriorate the male fly's performance in catching a target during extremely virtuosic chasing flights and appears not even to affect its flight style. In particular, it does not affect the fly's angular velocity as might be expected by the additive and the efference copy scheme. Rather, it is concluded that during chasing the optomotor system is largely suppressed. This result is extraordinary as it reveals the high efficiency of the blowfly chasing system to successfully cope with situations that in several other systems deteriorate performance.

\section{MATERIALS AND METHODS}

All experiments were performed with several sets of about 20 blowflies (Lucilia sp.). Each set was used for several days to conduct all types of behavioral experiments with the same animals. The flies (6- to 10-day-old) were released in a cylindrical flight arena (diameter: $0.4 \mathrm{~m}$, height $0.67 \mathrm{~m}$ ) made of clear Perspex; the ceiling was homogeneously white. The arena was illuminated by boards of green LEDs that were used as a panoramic stimulus (see below). Two tungsten light heads (DLH4, $150 \mathrm{~W}$, Dedo Weigert Film, Germany) additionally illuminated the arena from below. To ensure that these lamps did not much reduce the contrast of the visual stimulus and because red light is almost invisible for blowflies (Hardie, 1985), both lamps were fitted with dichroic red-light filters (DFCOL2R, Dedo Weigert Film, Germany). The temperature in the flight arena ranged between $20^{\circ} \mathrm{C}$ and $29^{\circ} \mathrm{C}$.

The upper $0.32 \mathrm{~m}$ of the flight arena was surrounded almost completely (see below) by a cylindrical stimulus device that consisted of two rows of ten circuit boards each, stacked over each other. Each board contained 48 columns and 30 rows of LEDs (Toshiba TLG234P, $2.5 \times 5 \mathrm{~mm}^{2}$, emitted wavelength $565 \mathrm{~nm}$ ). Each column could be switched on and off independently. The horizontal angular extent of each LED amounted to approximately $0.7^{\circ}$ as seen from the center of the arena at the same height of the respective LED. The time until an LED reached a constant luminance value after switching it on or off was $20-50 \mu$ s. The device was programmed to generate apparent motion of a vertical grating with a spatial wavelength of $30^{\circ}$ (corresponding to a spatial frequency of 0.033 cycles per degree) and $85 \%$ contrast. This grating was either stationary or rotated clockwise or counter-clockwise at $45 \%$ s or $365^{\circ} / \mathrm{s}$. These pattern velocities corresponded to temporal frequencies of $1.5 \mathrm{~Hz}$ and $12.2 \mathrm{~Hz}$ of the drifting grating. Generating one frame, i.e. addressing all groups of LED-columns serially, took approximately $370 \mu \mathrm{s}$. The cylindrical LED-array spanned $330^{\circ}$ in azimuth and approximately $75^{\circ}$ in elevation as seen from the center of the arena. $30^{\circ}$ of the cylinder were left open for the lateral camera to see the inside of the flight arena.
A black sphere (diameter: $5 \mathrm{~mm}$ ) was used as a chasing target ('dummy fly'). If the dummy was fixated by the chasing fly at a distance of $50 \mathrm{~mm}$ it had an angular size of approximately $6^{\circ}$, i.e. much larger than the interommatidial angle measured for frontally looking ommatidia (Land and Eckert, 1985). The sphere was attached to the tip of a translucent perspex stick (length: $65 \mathrm{~mm}$ ) that was fixed to a non-transparent, white circular disk (diameter: $0.395 \mathrm{~m}$ ) which could be rotated horizontally directly beneath the top end of the arena (vertical distance from upper rim of arena $45 \mathrm{~mm}$; total vertical distance of the target from the upper rim of the arena $0.11 \mathrm{~m}$ ). In the following the white disk will be referred to as 'ceiling' of the flight arena. The dummy was moved clockwise on a circular track (radius: $80 \mathrm{~mm}$ ) at a speed of $1 \mathrm{~m} / \mathrm{s}$ (i.e. about $700 \%$ s), which is well within the speed range of flies. The dummy and the visual background, generated by the LED-array, could be moved individually or in combination.

Flying flies were filmed with two orthogonally arranged digital high speed cameras (MotionPro 500, Redlake, San Diego, CA, USA, spatial resolution: $1024 \times 1024$ pixels $^{2}$ ) at a sampling rate of $250 \mathrm{~Hz}$. One camera viewed the upper part of the arena from the side through the gap of the LED array. The other camera was placed underneath the arena and covered the entire arena. For both views, the 2D positions of the fly and - if present - of the target were determined frame by frame with custom-made software. The longitudinal body axis orientation of the fly was determined from the bottom view only (for details of the procedures see Lindemann, 2006). Briefly, for both camera views a background image was calculated as the mean of 10 frames from different parts of a movie. This background image was subtracted from every frame, yielding difference images. In principle, difference images highlight moving objects and suppress all stationary items in the image. Unfortunately, the output of the camera chip suffers from pixel noise, and the noise pixels introduce false object pixels which have to be eliminated from the difference images. The difference images were binarized employing a user-defined threshold above noise level. The program seeks for connected object regions in the binary image (segmentation) and prunes the resulting region set by testing certain criteria such as the size (number of pixels constituting the region) and form of the region (cf. roundness). The position of the fly or dummy is assumed to correspond to the center of gravity of a respective region and its orientation to the orientation of its major axis. Because the position and orientation of the fly (region) are evaluated from a large number of pixels in the binary image (typically well above $100)$, errors are small in general.

Knowing the relative positions of the two cameras, 2D image coordinates were transformed into an orthographic 3D coordinate system (Zeil, 1983; Boeddeker et al., 2003). To assess methodological errors, the position and orientation of a perched fly was reconstructed. The apparent yaw velocity caused by orientation errors had a standard deviation of $45 \%$ s. Due to the high sampling rate of the cameras ( $250 \mathrm{fps})$ this standard deviation corresponds to a change of the estimated fly orientation between two consecutive frames of only $0.18^{\circ}$. The position error that is caused by distortions caused by the camera optics, increased with increasing eccentricity of the fly in the flight arena, but was always below $2 \mathrm{~mm}$. 
These data allowed the analysis of the flight trajectories with respect to the fly's yaw velocity (calculated from the changes in yaw angle, as determined from the data of the bottom camera, within $4 \mathrm{~ms}$ time intervals), forward velocity (calculated from the changes in $3 \mathrm{D}$ position within $4 \mathrm{~ms}$ time intervals), turning frequency, the distance to the target and the deviation angle of the target direction from the fly's body long axis orientation. This deviation angle approximates the retinal error angle of the target quite well, since the yaw angle of the head deviates only by few degrees from the orientation of the body length axis, and the roll angle does not change much during free-flight maneuvers despite considerable body roll during saccadic turns (van Hateren and Schilstra, 1999). The reconstruction of the 3D-trajectories and all further data analysis was done in Matlab 6.5 (Mathworks, Natick, MA, USA).

For the analysis of the time-structure of the flight trajectories only those flights were used that met certain criteria. (1) Optomotor and cruising flights lasted for at least $150 \mathrm{~ms}$. (2) Chases were classified according to whether the target was caught ('C-chases') or pursued ('P-chases') without catching it (Boeddeker et al., 2003). To ensure that males did not coincidentally fly for some time in the same direction as the target, but really chase it, flights were classified as P-chases only if the male followed the target for at least one lap of the dummy (i.e. for at least $510 \mathrm{~ms}$ ).

To evaluate the frequency of yaw turns executed during flights, it had to be defined which fluctuations of the yaw velocity profile are classified as turns. Although the yaw velocity traces change continuously, turns were defined by applying threshold operations. First, the mean angular velocity was subtracted from the angular velocity trace. In general, turns were detected, if the angular velocity exceeded a certain threshold and fell below this threshold after some time. Rightwards turns corresponded to positive thresholds, leftwards turns to negative ones. During chases and optomotor following the absolute value of the angular velocity frequently did not immediately fall down again to the mean angular velocity, but started increasing again from a more elevated level to reach a second peak. This peak was counted as a separate turn if the angular velocity decreased below one of five thresholds of increasing absolute size $(150 \% \mathrm{~s}, 300 \% \mathrm{~s}, 500 \% \mathrm{~s}, 800 \%$ s, and $2000 \% \mathrm{~s})$. For instance, the angular velocity may increase monotonically from its mean value to a value above the threshold of $500 \%$ s and then fell down below the threshold of $300 \%$, before it increased again to reach a second peak above the threshold of $800 \%$ s. In this case, two turns were counted. However, if the angular velocity did not decrease between the two peaks below the threshold of $300 \%$, only one turn was counted. The lowest threshold was chosen to be above three times the standard deviation of the methodological velocity error $\left(45^{\circ} / \mathrm{s}\right.$, see above).

\section{RESULTS}

Three distinct flight behaviors of blowflies were analyzed as well as their interactions, i.e. chasing behavior, optomotor following and, as a reference, cruising flight. A small black sphere moving in the upper part of the flight arena served as a target for chasing behavior. The flight sequences were recorded with a pair of highspeed cameras in a cylindrical flight arena surrounded by a grating pattern that was either stationary or moved horizontally at different velocities in either direction.

\section{DIFFERENT FLIGHT BEHAVIORS}

The term cruising flight is used here for flights which do not have an obvious goal and are not elicited by changes in the environment, for instance, by a moving target or the background. The dynamical features of cruising flights are distinguished by a series of rapid changes in the orientation of the body long axis (Figure 2Ai). The angular velocities generated during these rapid turns may reach frequently more than $2500 \%$ (Figure 2Aii). These rapid body turns exhibited during cruising flight are called - by analogy to rapid human eye movements - saccades (Collett and Land, 1975; Schilstra and van Hateren, 1999; van Hateren and Schilstra, 1999). Between saccades the orientation of the fly's body long axis remains relatively stable.

The behavior of male flies dramatically changes when a black sphere mimicking a female fly is set into motion. Such a female dummy, even when moving at constant velocity $(700 \%$ s) on a circular track, is pursued from below and behind. During chasing flights, the male frequently flies slightly outside the track of the target (Figure 2Bi). We classified the chases by their catching success (Boeddeker et al., 2003): In successful chases the target was caught after short time ('C-chases'; mean duration $=371 \mathrm{~ms}$, $\mathrm{STD}=172 \mathrm{~ms}, n=50$ ). In unsuccessful chases, the target was pursued for at least one lap of the target $(510 \mathrm{~ms})$ without catching it; the fly either approached but missed the target, or it gave up chasing and retired. While pursuing the target in front of a stationary background, the mean angular velocity of the fly is close to that of the target (Figure 2Bii). The fly continuously changes its yaw orientation and performs relatively smooth body turns (compare with body saccades during cruising flight; see above) of varying amplitude. As these yaw velocity fluctuations are not as transient and rapid as the saccades found during cruising flight, chasing behavior of blowflies has been concluded to be mediated by a smooth control system (Boeddeker and Egelhaaf, 2003, 2005; Boeddeker et al., 2003). Relatively large turning velocities are frequently observed at the beginning of a chase when the fly makes an initial turn towards the target and at the end of the chase when the fly tends to orient itself almost orthogonally to the target's direction of movement. Because we want to concentrate on the flight characteristics during on-going pursuit, these large turns were not included into the detailed quantitative analysis.

The saccadic flight style of blowflies appears to be abandoned not only when males chase moving targets, but also when the entire background is moving. When the vertical grating surrounding the flight arena rotated horizontally at a constant velocity either slowly $\left(45^{\circ} / \mathrm{s}\right)$ or fast $\left(365^{\circ} / \mathrm{s}\right)$, the flies tended to follow the visual wide-field motion on roughly circular tracks by continuous body rotations. In this way flies tend to reduce the retinal slip velocity induced by the imposed wide-field motion (Figure 2Ci). This so-called optomotor following is generally assumed to compensate for asymmetries in the animal's sensory and motor systems during locomotion (reviews: Wehner, 1981; Collett et al., 1993). Because the fly follows more or less closely the movements of the background, its mean yaw velocity is close to the angular velocity of the background and the fluctuations around this mean are smaller than many saccades during cruising flights (Figure 2Cii). 

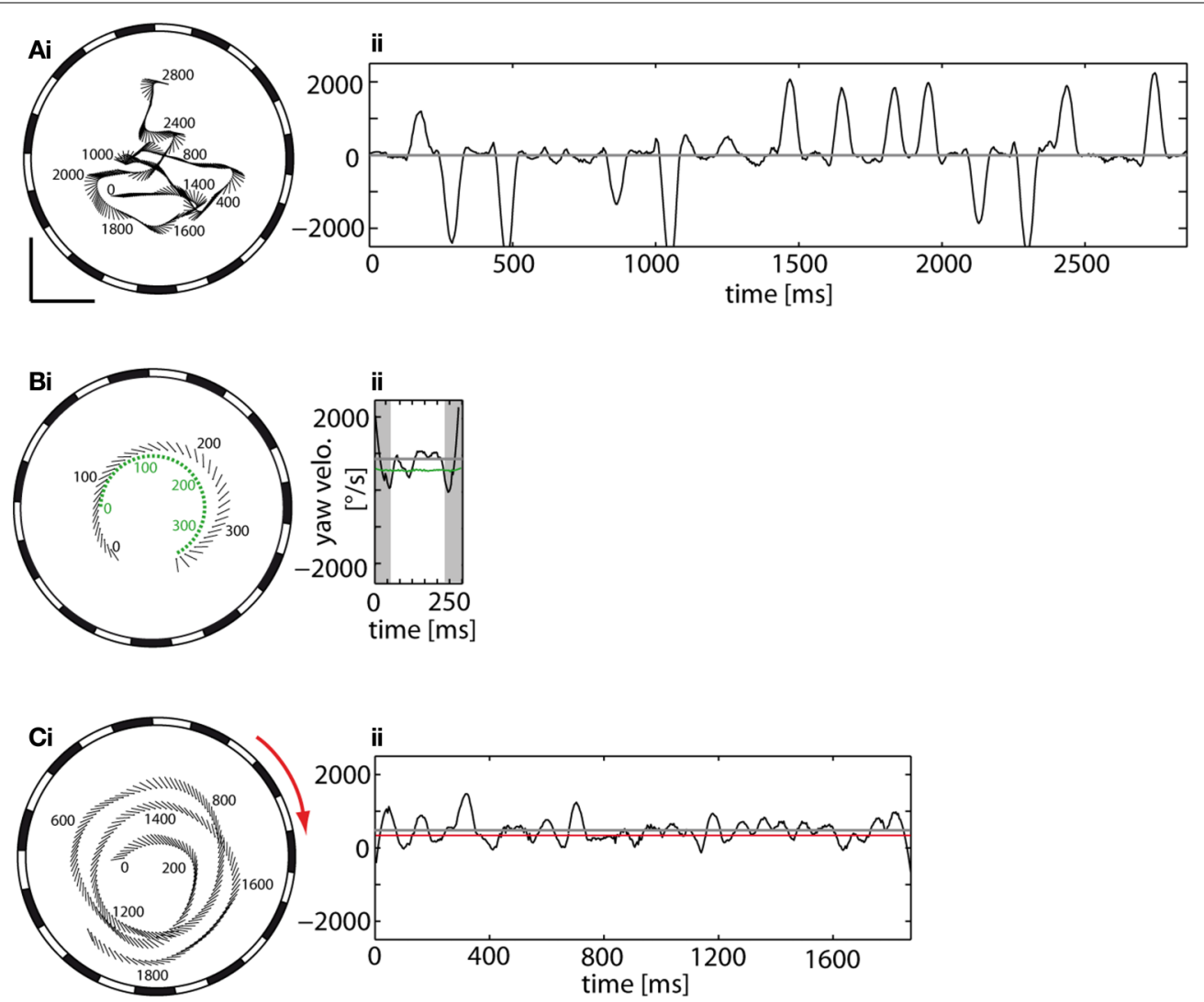

FIGURE 2 | Flight trajectories of male flies, as seen from below, and time-dependent yaw velocities of (A) a cruising flight, (B) a chasing flight and (C) an optomotor flight. (Ai) Flight trajectory of a male fly cruising in the arena that is surrounded by a stationary background (vertical grating). Numbers denote time (in $\mathrm{ms}$ ) with respect to the beginning of the trajectory. (Aii) The yaw velocity profile of the fly reflects the saccadic flight style characteristic of cruising flights. Between the turns exceeding $2500 \%$ are time intervals with little or no rotation. (Bi) Flight trajectory of a fly (black) chasing the target (green) which moved on a circular track. The position (centroid) of the fly is depicted by a dot, the orientation of its body long axis by the line. The flight arena is surrounded by a

The differences in flight style as shown in Figure 2 for examples of cruising behavior, chasing and optomotor following were quantified across different flies by evaluating flight parameters, such as the average yaw velocity, the peak yaw velocity deviations during turns from the average yaw velocity, the forward velocity as well as the turning frequency (Figure 3).

Both the mean yaw velocity and the peak yaw velocity deviations from average yaw velocity reflect the flies' flight mode (Figure 3A). During cruising flights the average yaw velocity is close to $0 \% \mathrm{~s}$, although male blowflies perform saccadic turns with often high peak velocities (Figure 2Aii). This is because during the intersaccadic intervals yaw velocity is close to 0 (Wagner, 1986b,c; Schilstra and van Hateren, 1999; van Hateren and Schilstra, 1999) and saccadic turns are distributed approximately equally in both directions during cruising flight. Thus, positive and negative velocities may occur with an approximately equal share (Trischler, 2009).

During optomotor following flies tend to turn according to the direction of the moving background. The average yaw velocity is increased as compared with that during cruising flights, i.e. to stationary background. Same plotting conventions as in Ai. (Bii) The yaw velocity of the fly changes continuously and relatively slowly while pursuing the target which moves on a circular path at $700 \%$ (green line). The mean yaw velocity of the fly is near $1000 \%$ (gray line). The initial and the final turn (shaded regions) were excluded from the quantitative analysis of flight parameters (see Materials and Methods). (Ci) Flight trajectory of a fly during optomotor following of a vertical grating moving clockwise (red arrow) at $365 \%$ s. Same plotting conventions as in Ai. (Cii) The yaw velocity of the fly fluctuates around its mean of $528 \%$ (gray line) which is somewhat larger than the velocity of the background (365\%; red line). Scale bars: $100 \mathrm{~mm}$. Note the different time axis scaling in (Aii,Cii).

$279^{\circ} / \mathrm{s}$ during slow and to $426 \%$ during fast background rotation (Figure 3A). Given that the background angular velocity amounted to $45 \%$ and $365^{\circ} / \mathrm{s}$, respectively, this indicates the somewhat paradoxical situation of a closed-loop optomotor gain, as given by the ratio of the angular velocity of the fly and that of the background (Collett, 1980), of more than 1 . Note, however, that the flies do not only rotate under our experimental conditions as is implicitly assumed when estimating the optomotor gain, but that they also translate. Combined rotation and translation results in complex optic flow patterns on the two eyes depending, apart from the current motion vector of the animal, on its position and orientation in the flight arena. In any case, during optomotor following the peaks of the angular velocity fluctuations around the average yaw velocity are much smaller than during cruising (compare also Figure 2Aii with Figure 2Cii). Since during optomtotor following the flies flew in the upper part of the arena (on average $115 \mathrm{~mm}$ below its ceiling), mainly regions below the equator of the eyes were stimulated by wide-field motion. This finding indicates, in accordance with a previous study on tethered flying flies that optomotor following can also be elicited in the equatorial 



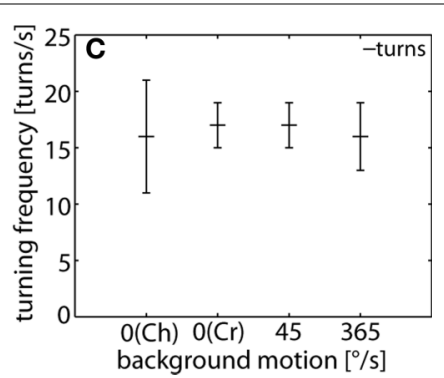

FIGURE 3 | (A) Yaw velocity, (B) turning frequency and (C) forward velocity during chases ( $\mathrm{Ch}$; stationary background), cruising flights ( $\mathrm{Cr}$, stationary background) and optomotor following induced by a slowly $\left(45^{\circ} / \mathrm{s}\right)$ and fast $(365 \%)$ moving background. Data obtained from chasing flights represent the averages over $\mathrm{C}$ - and P-chases. (A) Time averaged yaw velocities $( \pm S D)$ of a sample of flies (black) and mean peak yaw velocity deviations of the yaw velocity from its respective mean value $( \pm S D)$ of the same sample of flies (green). The flies' mean yaw velocity is near $900 \%$ for males pursuing a target that moved at $700 \%$ (asterisk). In cruising flights, the mean yaw velocity is close to $0 \%$. In optomotor flights the fly's mean yaw velocity increases with increasing background velocity. (B) The average turning frequency ( \pm SD) is relatively similar for chases, optomotor flights and cruising flights. (C) Time averaged forward velocity $( \pm S D$ ) of a sample of flies (black) and the mean standard deviations $( \pm S D)$ of the corresponding velocity fluctuations around the respective time averaged values (red). The fly's mean forward velocity is around $1.2 \mathrm{~m} / \mathrm{s}$ while chasing the target moving at $1 \mathrm{~m} / \mathrm{s}$ (asterisk). The flies' mean forward velocity is around $0.4 \mathrm{~m} / \mathrm{s}$ in a stationary environment during cruising flights; it increases with increasing background motion velocity. Cruising flights: $n=10$, total flight time $(\mathrm{TFT})=20804 \mathrm{~ms}$; optomotor flights at $45 \% \mathrm{~s}(365 \% \mathrm{~s}): n=4(n=8)$, TFT $=1820 \mathrm{~ms}$ (TFT $=5544 \mathrm{~ms}$ ); chases: $n=17, \mathrm{TFT}=4740 \mathrm{~ms}$. and ventral parts of the visual field (Borst and Bahde, 1987). In a similar way, stimulation of the ventral eye is needed in bibionid flies to evoke optomotor responses, whereas only the dorsal eyes promote male-specific visually guided behavior (Zeil, 1983).

During chasing after the dummy, the males turn continuously at high velocities. Only rarely the yaw velocity assumes $0 \% \mathrm{~s}$ for a longer time. By contrast, during cruising flights the fly does not turn much between saccades. The peak angular velocity deviations from average yaw velocity are again much smaller during chasing than is characteristic of cruising flights. Hence, when pursuing a target that moves on a circular path and when following a continuously moving optomotor stimulus, blowflies follow the respective visual stimulus by changing their body orientation much more smoothly than during saccadic cruising flights.

The average forward velocity during cruising in our flight arena amounts to $0.4 \mathrm{~m} / \mathrm{s}$. This value increases during optomotor following with increasing background velocity to $0.55 \mathrm{~m} / \mathrm{s}$ and $0.8 \mathrm{~m} / \mathrm{s}$ during slow and fast background motion, respectively (Figure 3B). This finding indicates that optomotor following under free-flight condition is not only accomplished by turning responses but, in addition, by adjusting the translation velocity. While pursuing a target moving at $1 \mathrm{~m} / \mathrm{s}$ the chasing fly has an average forward velocity of $1.18 \mathrm{~m} / \mathrm{s}$ (Figure 3B). It is remarkable that during chasing, males can triplicate the forward velocity adopted during cruising flights in the same flight arena. This finding is in accordance with previous conclusions that the translational velocity during chasing is increased until a critical retinal target size is reached (Boeddeker and Egelhaaf, 2003; Boeddeker et al., 2003). Independent of the exact environmental conditions and the flight mode, the velocity fluctuations around the mean forward velocity range between $0.11 \mathrm{~m} / \mathrm{s}$ and $0.18 \mathrm{~m} / \mathrm{s}$. Hence, at least during optomotor following and during chasing the variations of forward velocity are small relative to the respective mean velocities.

Blowflies execute on average 16-18 turns/s during cruising, optomotor following and chasing (Figure 3C), although the peak velocity of turns might differ tremendously for the different behaviors. This finding indicates that the number of turns is quite independent of the flight behavior and of the environmental conditions, although the characteristics of the turns differ considerably.

\section{CHASING BEHAVIOR DURING BACKGROUND MOTION}

Chasing behavior and optomotor following that were investigated separately, so far, may interfere with each other under normal flight conditions. When the male fly turns during a chase towards its moving target, the retinal image of the background inevitably moves in the opposite direction on the fly's retina and, if it is textured, may activate optomotor following. Optomotor following may then counteract fixation of the target mediated by the chasing control system. Consequently, the two control systems may be in conflict with each other.

To elucidate the potential impact of the optomotor system on chasing behavior, we conducted a set of further behavioral experiments. Since it is hardly possible to eliminate any background textures and thus some sort of background stimulation during self-motion, we employed an alternative approach and presented simultaneously a dummy fly moving at $700 \%$ on a circular track and wide-field motion of the vertical grating surrounding the flight arena. This background grating moved either slowly $\left(45^{\circ} / \mathrm{s}\right)$ or fast $\left(365^{\circ} / \mathrm{s}\right)$ in the same direction as the target ('positive background motion') or in the opposite direction ('negative background motion'). As reference condition, the background was held stationary. We analyzed the potential impact of background motion (1) on catching success of the pursued target and (2) on various relevant flight parameters, such as turning and translational velocity as well as turning frequency but also the error angle under which the target is fixated by the chasing fly. During chasing, when the pursuing fly is close to the dummy target the average altitude of chasing fly is $50 \mathrm{~mm}$ below the dummy track and, thus, about $115 \mathrm{~mm}$ below the ceiling of the flight arena. Then the optomotor stimulus is seen - depending on the pitch angle of the head-mostly in eye regions below the equator. The stimulated eye region thus overlaps largely with that that was stimulated, on average, during optomotor following (see above). 
To assess a potential impact of background motion on the catching success of chasing flies we determined the average number of catches occurring within 250 independent $40 \mathrm{~s}$ time-windows while 20 male flies were in the flight arena. These 20 male flies, on average, caught the dummy between 14 and 17 times per timewindow when the background was stationary or moved slowly in either direction (Figure 4A). Even at high background velocities male flies were still able to catch their target frequently. However, the catching frequency decreased by about one third to just below 10 catches per time-window. One potential reason for the decrement in catching success is a reduction in the readiness of flies to fly at all during fast background motion, rather than the performance of the chasing system. This issue was addressed by determining the average number of flies that flew simultaneously at each instant of time for the different background conditions (Trischler, 2009). Since flight sequences usually last for only few seconds, many different flies flew during the counting period even if the mean number of flying flies at each instant of time ranges, on average, only between 5 and 2. The number of flies flying simultaneously at any time in the arena was similar in the stationary and slowly moving arena, but is approximately halved when the background pattern moved fast (Figure 4B). Hence, fast background motion obviously diminishes the readiness of flies to fly. This result suggests that the catching success does most likely not deteriorate as a consequence of an interference of background motion with the chasing system after the fly has initiated a chase. Nevertheless, these data do not exclude that background motion influences the flight behavior during the chase in a more subtle way.
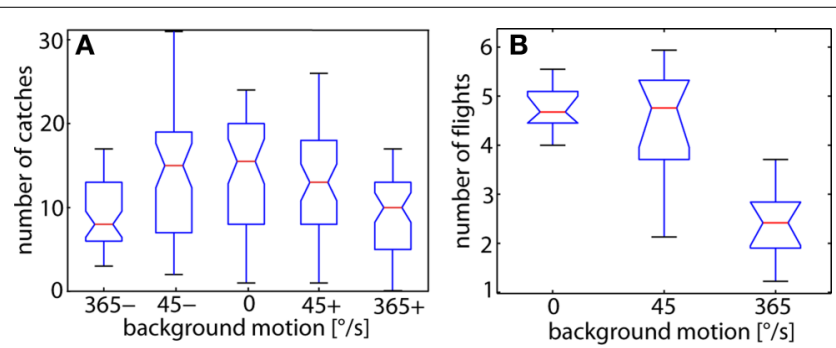

FIGURE 4 | (A) Box-Whisker plots of the number of catches during time windows of $40 \mathrm{~s}$. The target was moving at $700 \%$ s under various background conditions. For each background condition the catches were counted in 50 time-windows (each lasting $40 \mathrm{~s}$ ). The background was either moving slowly $\left(45^{\circ} / \mathrm{s}\right)$ or quickly $\left(365^{\circ} / \mathrm{s}\right)$ in the same (positive sign) direction as or in the opposite (negative sign) direction to the target. As a reference, the background was held stationary $\left(0^{\circ} / \mathrm{s}\right)$. The catching frequency is significantly decreased at high background velocities with reference to the number of captures obtained while the background was stationary (Wilcoxon signed rank test; $P<0.01$ ). Catches: $n=2954$. (B) Number of flies flying simultaneously at any time in the arena under the three different background conditions. The background was either stationary $(0 \%$, reference), or moved at $45 \%$ s or at $365 \%$ s (positive and negative direction values pooled). The number of flies flying was determined during each $1 \mathrm{~s}$ time bin of a 30-s time-window and averaged over the 30-s time-window. The averages shown in the figure are obtained from 10 trials. Since flight sequences usually last for only few seconds, many different flies flew during the counting period even if the mean number of flying flies at each instant of time ranges, on average, only between 5 and 2 . The number of flights was found to be significantly smaller at the high background velocity with reference to the stationary background (Kruskal-Wallis test; $P<0.01$ ). $1 \mathrm{~s}$ time-bins: $n=1800$.
To quantify a possible impact of wide-field motion on the fine structure of the chasing flights, we evaluated the temporal structure of chasing trajectories. The analysis was done separately for $\mathrm{C}$ - and P-chases. According to predictions based on both the additive and the efference copy scheme of how pursuit and optomotor following might interact, background motion should affect the turning velocity of the chasing fly, although these predictions take only the rotational degree of freedom of movement into account. In contrast to expectations, the average yaw velocity during both $\mathrm{C}$ and $\mathrm{P}$-chases ranges between $800 \% \mathrm{~s}$ and $900 \%$ for all background conditions (Figures 5A,B). The results for the $\mathrm{C}$ - and $\mathrm{P}$-chases are similar, although $\mathrm{P}$-chases are less variable than $\mathrm{C}$-chases. For both types of chases the angular velocity did not depend consistently on

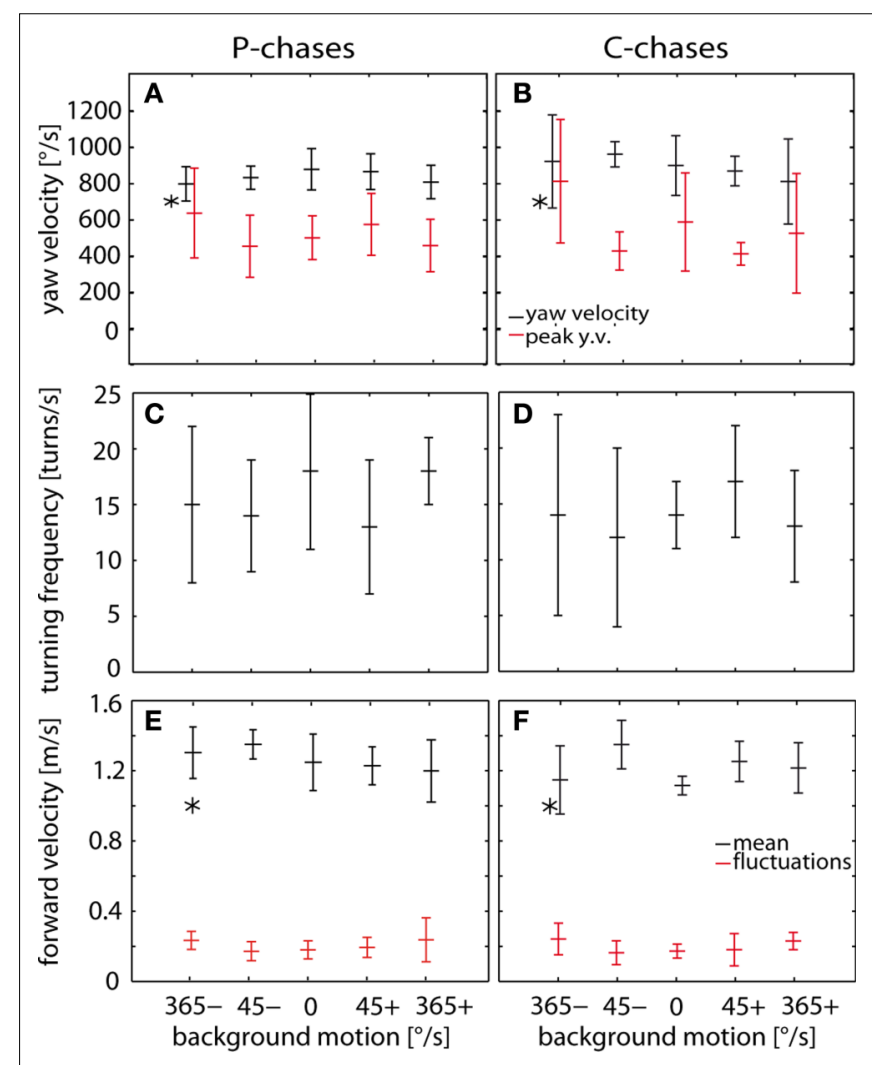

FIGURE 5 | Yaw velocity, forward velocity and turning frequency determined separately for $\mathbf{C}$ - and $\mathbf{P}$-chases for five different background conditions: the grating was held stationary as a reference $(0 \% / \mathrm{s})$ or the grating either moved slowly $\left(45^{\circ} / \mathrm{s}\right)$ or fast $\left(365^{\circ} / \mathrm{s}\right)$ in the same direction as the target (positive sign) or in the opposite direction to the target (negative sign). (A,B) Time-averaged ( \pm SD) yaw velocity (black) and mean $( \pm S D)$ deviation peaks from average yaw velocity (red) determined for P-chases (A) and C-chases (B). The target moved at 700\% (asterisk). (C,D) Time-averaged $( \pm S D)$ forward velocities (black) and the average $( \pm S D)$ velocity fluctuations (red) determined for P-chases (C) and C-chases (D). The flies followed the target that moved at $1 \mathrm{~m} / \mathrm{s}$ (asterisk). (E,F) Overall average ( \pm SD) turning frequency determined for P-chases (E) and C-chases (F). Mean and standard deviation were calculated across flies. $0 \%$ : C-chases $n=7$, total flight time $(T F T)=1896 \mathrm{~ms}$; P-chases $n=10$, TFT $=2844 \mathrm{~ms} .+45 \%$ : C-chases $n=7$, TFT $=2288 \mathrm{~ms}$; P-chases $n=8, \mathrm{TFT}=2492 \mathrm{~ms} .-45 \%$ : C-chases $n=4$, TFT $=748 \mathrm{~ms}$; P-chases $n=8$, TFT $=3912 \mathrm{~ms} .+365 \%$ $\mathrm{s}$ : C-chases $n=9, \mathrm{TFT}=2788 \mathrm{~ms}$; P-chases $n=7, \mathrm{TFT}=3108 \mathrm{~ms} .-365 \%$ : C-chases $n=9$, TFT $=2928 \mathrm{~ms}$; P-chases $n=9$, TFT $=4024 \mathrm{~ms}$. 
background velocity. Moreover, background motion does not affect the peaks of the fluctuations around the average yaw velocity during both $\mathrm{C}$ - and P-chases (Figures 5A,B). The frequency of turns ranges in $\mathrm{C}$ - and $\mathrm{P}$-chases for all background conditions between 12 and 18 turns per second in $\mathrm{P}$-chases and in $\mathrm{C}$-chases without consistent dependence background motion (Figures 5C,D).

Since background motion in a non-chasing situation was found to affect not only the rotational but also the translational velocity of flies, we also scrutinized potential changes in translational velocity induced by background motion during chasing behavior. Again, wide-field motion does not affect systematically the translation velocity during both $\mathrm{C}$ - and P-chases. Moreover, in both chasing modes the velocity fluctuations are small around average velocity (on average around $0.2 \mathrm{~m} / \mathrm{s}$ ) and increase only slightly with increasing background velocity (Figures 5E,F).

To assess potential consequences of wide-field motion on the final velocity before catching the target during $\mathrm{C}$-chases, the time course of the flies' decreasing distance to the target was determined for the last $200 \mathrm{~ms}$ of successful chases. Since the translation velocity of flies is much influenced by background in the non-chasing flight mode, one could imagine that a chasing fly is retarded from catching the target by negative background motion and pushed towards the target by positive background motion, respectively. Although there is some variability in the time course of distance reduction with background velocity, no consistent dependence on background velocity is observed (Figure 6A). The standard errors of the time-dependent distance curves overlap widely, suggesting that even the flies' performance during the final flight phase before catching the target is not affected by background motion.

Since the error angle of the target on the chasing male's retina was concluded to be one visual parameter controlling chasing behavior (Boeddeker and Egelhaaf, 2003; Boeddeker et al., 2003), we investigated whether it is affected by background motion. Again, the error
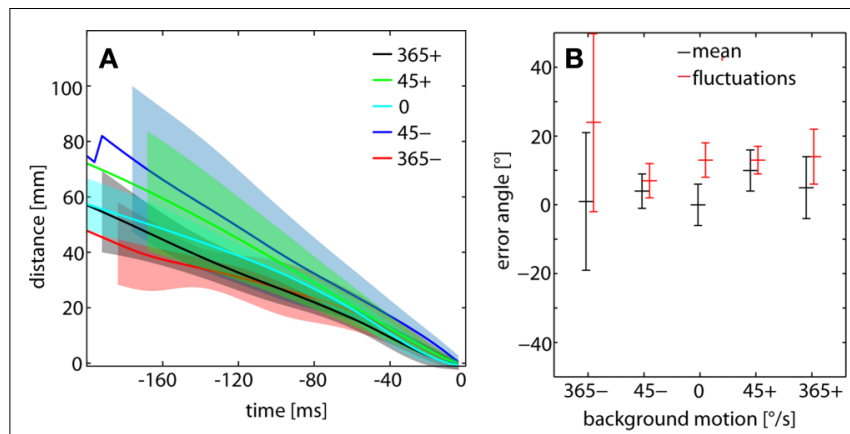

FIGURE 6 | (A) Distance between chasing fly and target and (B) error angle of the target determined for $\mathrm{C}$-chases for the different background conditions. (A) Mean time course of the flies' distance to the target for the last $200 \mathrm{~ms}$ before catching the target. The data were aligned to the end of the flight episode (i.e. the catch represents time $0 \mathrm{~ms}$ ). Colored lines represent the mean time course; similarly colored shaded areas depict the respective standard error of the mean. For the sake of clarity, these areas are shortened differentially. Mean and standard deviation were calculated across flies. $n=36$ chases, total flight time $=7200 \mathrm{~ms}$. Color code of background conditions given in the inset. (B) Mean error angle (black) obtained from a sample of flies. The average (time-dependent) fluctuations of the target on the eyes (red) range between $7^{\circ}$ and $24^{\circ}$. Chases: $n=36$, total flight time $=10648 \mathrm{~ms}$. angle is evaluated only for C-chases which eventually terminated with catching the target. The target is, on average, seen in the frontal visual field and only slightly displaced - on average by $0^{\circ}$ and $+10^{\circ}$ - into the direction of target motion (Figure 6B). Does background motion have an impact on the target's error angle during a blowfly's chase? There is a slight increase in the fixation error at slow background velocities. However, this tendency is no longer obvious at high background velocities (Figure 6B). The fluctuations of the error angle are, on average, somewhat larger at high background velocities. These values range between $7^{\circ}$ and $24^{\circ}$, which indicates that the target is still fixated within the frontal part of the visual field. Altogether, we do not find indications of pronounced and systematic dependencies of chasing performance on background velocity.

\section{DISCUSSION}

In behavioral experiments we examined two flight control systems of blowflies: the chasing system of males and the optomotor system which is implemented in both sexes, as well as the potential interactions of the control systems. Three findings are particularly striking. (i) The characteristic saccadic flight and gaze strategy as is a distinguishing feature of cruising flights is largely abandoned when the entire visual surroundings move around the fly; then flies tend to follow the moving pattern in a relatively continuous and smooth way. (ii) When male flies engage in following a small target, they also employ a smooth pursuit strategy (see also Boeddeker et al., 2003). (iii) The performance and dynamical characteristics of chasing are not much affected when the background moves in either the same or in the opposite direction as the target. Hence, optomotor following is overridden by the chasing system and does not much deteriorate chasing performance. It is concluded that during chasing after a moving target the optomotor system is largely suppressed.

Following moving targets is highly relevant for male blowflies, as they have to catch flying females in order to reproduce. In contrast, following moving wide-field patterns will occur in nature only rarely. Normally, wide-field motion on the eyes is only induced by the animal's own movements. Thus optomotor following is thought, under normal flight conditions, to stabilize the flight against unintended disturbances that cause asymmetries in the optic flow across the two eyes. A saccadic flight and gaze strategy as is a characteristic of many insects' cruising flight behavior (e.g. Wagner, 1986a,b,c; Land and Collett, 1997; Schilstra and van Hateren, 1999; van Hateren and Schilstra, 1999; Kern and Egelhaaf, 2000; Tammero and Dickinson, 2002; Mronz and Lehmann, 2008) is abandoned during chasing behavior and optomotor following (see also Mronz and Lehmann, 2008). There is evidence in blowflies that the translatory optic flow generated during the straight gaze segments between saccadic turns is extracted by the visual system to obtain spatial information about the environment (Kern et al., 2005, 2006; Lindemann et al., 2005, 2007; Karmeier et al., 2006 ). This information is no longer easily available during chasing behavior and optomotor following. It will be discussed below why this may not lead to severe problems for flies.

\section{PURSUIT OF A SMALL TARGET}

Chasing behavior of male blowflies belongs to the fastest visually guided behaviors found in nature. Generally, the chasing system is viewed as a feedback control system that minimizes deviations 
of the images of small objects from the midline of the visual field. The chasing blowfly keeps the retinal position of the target in the frontal field of view by predominantly smooth rotations about the vertical body axis. Thus, the average error angle of the target on the retina is small during chases (Figure 6; Boeddeker et al., 2003). The flies' forward and the yaw velocities are adjusted to the target's flight dynamics. Hence, under our experimental conditions, the average forward velocities of flies pursuing a quickly moving target $\left(700^{\circ} / \mathrm{s}\right)$ are increased threefold compared to the forward velocities during cruising flight in the same flight arena (Figure 3B). During chases, forward and yaw velocity are controlled by the angular size and the error angle of the target, respectively (Boeddeker and Egelhaaf, 2003, 2005). A set of sex-specific neurons in the third visual neuropil of the male blowfly's brain has been concluded to represent the neural substrate for target specificity during chasing (Hausen and Strausfeld, 1980; Gilbert and Strausfeld, 1991; Strausfeld, 1991; Wachenfeld and Hausen, 1993, 1994). On the basis of naturalistic stimuli it was found that at least one prominent neuron of this ensemble shows a distinct direction selectivity and complex nonlinear response characteristics to the joint occurrence of different visual parameters of the target including size, position and velocity and the changes of these parameters over time (Trischler et al., 2007).

A control system that guides visual pursuit of a small target by visually induced changes in gaze direction mediated by movements of the eyes, the head and/or the entire body, is not only a characteristic of male blowflies, but has been described in other insects, such as other fly species (e.g. Land and Collett, 1974; Collett, 1980; Boeddeker et al., 2003) praying mantids (Rossel, 1980), dragonflies (Olberg et al., 2000) and bees (Gries and Koeniger, 1996). Not surprisingly, neurons have also been found in hoverflies and dragonflies, which are extremely sensitive to small moving objects and virtually do not respond to background textures (Olberg, 1981; O'Carroll, 1993; Frye and Olberg, 1995; Nordström and O'Carroll, 2006; Nordström et al., 2006; Barnett et al., 2007). However, targets can be pursued also on the basis of other sensory cues, such as auditory information as is employed, for instance, during phonotactic orientation of some insect groups (e.g. Stout et al., 1987; Weber et al., 1987; Böhm et al., 1991).

Our conclusion that in blowflies chasing behavior is mediated by a smooth control system is in accordance with previous studies on chasing behavior of other fly species. Smooth chasing interrupted by body saccades, if the retinal image of the target moves outside the frontal part of the visual field, has been shown also for the small housefly Fannia canicularis (Land and Collett, 1974), for the hoverfly Syritta pipiens (Collett and Land, 1975) and for the dolichopodid fly Poecilobothrus nobilitatus (Land, 1993a). In contrast, a saccadic chasing system was concluded to account for chasing in the male housefly Musca domestica (Wagner, 1986b).

In primates, including humans, a smoothly moving target evokes a combination of smooth and saccadic eye movements (review: Orban de Xivry and Lefèvre, 2007) depending on the target speed and on the target displacement with respect to the fovea, similar as in chasing blowflies (Boeddeker and Egelhaaf, 2005). At low target speeds, the target is kept centerd in the fovea by slow eye movements that follow the target smoothly. When the target is too rapid at high target speeds and displaced outside the fovea, smooth pursuit is interrupted by saccades to center the target again (Boeddeker and Egelhaaf, 2005; review: Land, 1999). Smooth pursuit of primates plays its most important role in everyday life when fixating stationary objects during locomotion. Then the retinal image of the object can only be kept in the fovea, if it is pursued by smooth eye movements. In contrast to flies, that may fixate a rapidly moving target during their aerobatic chases for several seconds, the phases of smooth pursuit in primates are relatively short and require the eyes to turn by only some tens of degrees. Model simulations revealed that the smooth chasing system of blowflies may, as a consequence of inevitable time constants in the fixation controller, even generate the catch-up saccades of the head and the entire body if the target is displaced too rapidly on the pursuing fly's retina (Wagner, 1986b; Boeddeker et al., 2003). The model employed is somehow similar to the proposal of a kind of integrator in the pursuit system that becomes operative when the target cannot be fixated as a consequence of background motion and, after reaching a threshold, induces a fixation saccade (Kirschfeld, 1997). The smooth and the saccadic components of pursuit eye movements in primates are usually thought to be controlled by distinct neural systems. However, recent findings suggest that saccades and pursuit are two outcomes of a single sensorimotor process that aims at orienting gaze direction (De Brouwer et al., 2001, 2002; Gardner and Lisberger, 2001; Orban de Xivry and Lefèvre, 2007).

\section{OPTOMOTOR FOLLOWING}

Whenever an animal moves in its environment, the retinal images move continually across the eyes. This so-called optic flow is exploited by visual systems in various ways. Components of the optic flow are assumed to form an input to the optomotor control system. When freely flying flies are confronted with a large-field rotating environment, they compensate to some extent the rotation of the background (corresponding to an apparent unintended self-rotation) by turning responses in the direction of the visual motion stimulus (Figures 2C and 3A; e.g. Götz, 1968; Collett, 1980; Heisenberg and Wolf, 1984; Warzecha and Egelhaaf, 1996; Mronz and Lehmann, 2008). During optomotor following under freeflight conditions where the animal experiences a complex mixture of rotational and translational optic flow, depending on its own movements but also the 3D structure of the environment, both the yaw and forward velocity of blowflies are increased with increasing background velocity (Figure 3A). A similar result was obtained in Drosophila (Mronz and Lehmann, 2008) and the hoverfly Syritta where the yaw velocity increased roughly linearly with the slip speed of the pattern across the fly's retina (Collett, 1980). In addition, the optomotor stimulus influenced the translational velocity of both Drosophila (Mronz and Lehmann, 2008) and Syritta (Collett, 1980). It should be noted that the rotation of the arena in these experiments on free-flying flies does not only induce rotational optic flow on the eyes, but, since the animals are usually not in the center of the flight arena, also strong translational optic flow, depending for the different eye regions on the relative location of the animal in the arena. It has been concluded from behavioral experiments that the translational velocity of insects appears to be controlled quite generally by the translational optic flow component on their eyes even in stationary environments where the 
optic flow depends, apart from the animal's movement vector, on its distance to objects in the environment (Srinivasan et al., 1991; Kimmerle et al., 1996).

In primates (including humans), coherent wide-field motion as may be induced on the eyes when the animal rotates around its vertical body axis constitutes the input to the so-called optokinetic system which evokes following movements of the eyes. This optokinetic reflex serves a similar function as the optomotor response of insects: By counter-directed eye movements the optokinetic reflex compensates image slip induced by large-field motion, such as occurs during self-motion of the observer. Together with the vestibulo-ocular reflex, which provides fast compensation for head rotation, the optokinetic reflex helps to stabilize gaze when the head and the body move, such as during self-motion. The optokinetic reflex is characterized, like pursuit of small targets, by smooth eye movements intermitted by relocating saccades (Ilg, 1997).

\section{INTERACTION BETWEEN PURSUIT AND OPTOMOTOR CONTROL SYSTEM}

During turns towards a target, such as during chasing, the actively generated large-field image displacements may contain strong rotational components and, thus, activate the optomotor following system. This system may then try to stabilize the flight path by generating turns away from the pursued target. Thus, the two control systems may be in conflict with each other, and this might impair the chasing performance. Moreover, since the translational velocity of flies was shown to be affected by background motion, the performance of a fly chasing after a target might also be affected by the translatory component of background motion.

How do chasing male blowflies deal with the potentially conflicting situation? Obviously they do it very efficiently. Although the readiness of blowflies to fly at all decreases at high background velocities, neither the performance of catching the moving target deteriorates much when the background is moved artificially with various velocities in either direction, nor could we pinpoint any impact on the error angle under which the target is fixated in the frontal visual field and the time course of chasing flights. These findings are remarkable, since background motion on its own does not only affect the overall rotational and translational velocity of the fly, but also the dynamic structure of flights by evoking largely smooth optomotor following responses. Hence, wide-field background motion appears to reduce the likelihood of saccades to occur.

The robustness of blowfly chasing behavior against large-field retinal image displacements is in contrast to other systems mediating fixation and pursuit of small targets. Female flies fixate stationary and slowly moving objects (Virsik and Reichardt, 1976; Reichardt et al., 1983; Kimmerle et al., 1997; Duistermars et al., 2007). However, in contrast to the male chasing system, the retinal error angle under which the target is fixated increases in females when the background is continually displaced at a constant velocity (Virsik and Reichardt, 1976). Furthermore, in praying mantids smooth pursuit deteriorates in front of a textured background, and the animal often switches to a saccadic pursuit strategy (Rossel, 1980; Kral, 2003). Such a shift from a smooth chasing mode of operation to a saccadic mode as has been hypothesized for target fixation and pursuit in flies (Kirschfeld, 1997). Our findings, however, reveal that such a shift does not occur systematically even when the background is moving.
The interaction between wide-field motion and target pursuit has been also addressed in primates. Numerous studies indicate that target pursuit is clearly influenced by wide-field stimulation, although the details of the influences are diverse (Collewijn and Tamminga, 1984; Keller and Khan, 1986; Kimmig et al., 1992; Masson et al., 1995; Mohrmann and Thier, 1995; Spering and Gegenfurtner, 2007).

How can the extraordinary performance of male blowflies in chasing small moving targets even in front of a moving background be explained? A wide range of mechanisms have been proposed so far to account for the interaction of two, potentially conflicting, behavioral responses (Crapse and Sommer, 2008). Three such interaction schemes are widely discussed also with respect to insect behavior, the additive scheme, the efference copy scheme and the suppressive scheme (see Introduction, Figure 1). Can any of these schemes explain the interactions between the blowfly chasing system and optomotor following, and also the control system mediating the saccadic flight style when the animal is cruising around?

In order to explain by the additive scheme (see Figure 1A) that chasing performance is largely independent of background motion the gain of the chasing system had to be much larger than that of the optomotor system. In the hoverfly Syritta the gain of the chasing system differs from that of the optomotor system in a frequency dependent way: it remains constant within a broad range of tested frequencies, whereas the optomotor system responds strongly only at low frequencies (Collett, 1980). Female houseflies, Musca, revealed similar dynamic differences in its object-fixation system and the optomotor system (Egelhaaf, 1987; Egelhaaf et al., 1988). Here, the object-detection system shows its strongest responses to transient object movements, i.e. at high frequencies, whereas the optomotor system responds best to much lower frequencies due to low-pass filtering in the visual motion pathway (Hausen, 1982a,b; Egelhaaf, 1987; Egelhaaf et al., 1988; Hausen and Egelhaaf, 1989). Recently, dynamic properties of these two control systems resembling those of hoverflies and houseflies were also found in the fruit fly Drosophila (Sherman and Dickinson, 2003; Duistermars et al., 2007). With dynamic separation of the visual consequences of object fixation and of optomotor following, both systems can combine additively and still operate relatively independently in the different dynamic ranges. Dynamic separation could be a simple strategy to almost eliminate the unwanted optomotor influence on active turns, since the generation of rapid turns (head or body saccades) during object fixation may induce retinal motion of the environment beyond the dynamic sensitivity range of the optomotor system (Collett, 1980; Egelhaaf, 1987; Duistermars et al., 2007). Nonetheless, the additive scheme with a frequency separation of the chasing system and optomotor following cannot easily explain the chasing performance of male blowflies. This is because in our systems analysis, where the male flies chased a constantly moving target smoothly, the background, as a consequence, was also displaced smoothly in the opposite direction. If no other computational measures were taken by the fly's brain, the optomotor system would respond under these conditions very well to the large-field motion and, thus, may impede the chasing performance by compensatory yaw body turns.

Also the efference copy scheme (von Holst and Mittelstaedt, 1950) cannot account for the interaction between the chasing and the optomotor systems. The 'efference copy' which represents the 
expected image motion during a turn is subtracted from the actual signal. Only if the turn occurs correctly, no optomotor response is evoked, but if it is subject to additional input, the optomotor system will still detect this and respond accordingly. Hence, if the chasing fly does not only rotate, but also translates and, in particular, if the outside environment moves globally as in part of our experiments, it is hardly possible that the nervous system is able to predict the visual consequences of self-motion and compensates for it via an efference copy. This is because the animal has no a priori knowledge of the 3D-layout of the environment that affects the retinal image motion during translatory self-motion not to speak of external global image displacements. Thus, if the efference copy scheme would apply to the chasing of male blowflies, the error angle between the target and the chasing fly should be affected consistently by optomotor stimulation - in contrast to our experimental results.

From these considerations it appears to be most likely that the chasing commands control a gating system that reduces the gain of the optomotor system during chasing or even suppresses it completely, probably in a largely frequency independent way (Figure 1C). In this regard the chasing system is similar to the phonotactic system of the cricket which has also been concluded, despite earlier evidence in favor of an additive scheme (Stout et al., 1987; Weber et al., 1987; Böhm et al., 1991) to rely on a suppression of optomotor following during pursuit (Webb et al., 2004). During chasing behavior of blowflies not only optomotor following is suppressed, but also the saccadic flight and gaze style which is characteristic of cruising flight and is characterized by the alternation of rapid saccadic turns and straight flight segments with virtually no rotation between saccades.

\section{FUNCTIONAL CONSEQUENCES: NO SPATIAL INFORMATION IS AVAILABLE DURING CHASING}

If the interpretation were correct, that the saccadic flight strategy of blowflies is a means to facilitate the processing of spatial information during the translatory phase in the intersaccadic interval (Kern et al., 2005; Karmeier et al., 2006; Lindemann et al., 2007; review: Egelhaaf, 2009) it would be hard for the animal to obtain spatial information during chasing maneuvers as well as during optomotor following.

\section{REFERENCES}

Barnett, P. D., Nordström, K., and O'Carroll, D. C. (2007). Retinotopic organization of small-field-targetdetecting neurons in the insect visual system. Curr. Biol. 17, 1-10.

Boeddeker, N., and Egelhaaf, M. (2003). Steering a virtual blowfly: Simulation of visual pursuit. Proc. R. Soc. Lond., B, Biol. Sci. 270, 1971-1978.

Boeddeker, N., and Egelhaaf, M. (2005). Chasing behaviour of blowflies: a smooth pursuit system generates saccades. J. Exp. Biol. 208, 1563-1572.

Boeddeker, N., Kern, R., and Egelhaaf, M. (2003). Chasing a dummy target: smooth pursuit and velocity control in male blowflies. Proc. R. Soc. Lond., B, Biol. Sci. 270, 393-399.
Böhm, H., Schildberger, K., and Huber, F. (1991). Visual and acoustic course control in the cricket Gryllus bimaculatus. J. Exp. Biol. 159, 235-248.

Borst, A., and Bahde, S. (1987). Comparison between the movement detection systems underlying the optomotor and the landing response in the housefly. Biol. Cybern. 56, 217-224.

Collett, T.S. (1980). Angular tracking and the optomotor response. An analysis of visual reflex interaction in a hoverfly. J. Comp. Physiol. 140, 145-158.

Collett, T. S., and Land, M. F. (1975). Visual control of flight behaviour in the hoverfly Syritta pipiens L. J. Comp. Physiol. 99, 1-66.

Collett,T.S.,Nalbach,H.-O., andWagner,H. (1993). "Visual stabilization in arthropods," in Visual Motion and its Role in

Optomotor following of a moving environment does not occur in normal behavioral situations, as flies usually move in stationary environments. Optomotor responses are rather assumed to compensate for unintended rotations which may occur as a consequence of internal asymmetries of the animal (Strauss and Heisenberg, 1990; Collett et al., 1993; Hengstenberg, 1993; Kern and Egelhaaf, 2000). Slowly varying rotational disturbances are compensated most efficiently (Egelhaaf, 1987, 1989; Warzecha and Egelhaaf, 1996) in accordance with the view that optomotor course stabilization is destined to mainly balance the consequences of internal asymmetries of the animal. Hence, under normal conditions optomotor responses are supportive to ensure by feedback control a straight gaze during the intersaccadic interval.

During chasing, the situation is different because the animal is confronted with a moving target that is pursued very efficiently by a smooth control system keeping the target largely fixated in the frontal region of the visual field. In addition, during chasing saccades only appear to be generated as catch-up saccades when the target changes its direction too rapidly to be fixated by smooth following movements (Boeddeker and Egelhaaf, 2005). Hence, in contrast to cruising flight, during chasing the translational optic flow may be superimposed by a pronounced rotational component which makes it hard to extract distance information. How can the pursuing fly cope with this problem? This may indeed not be a problem at all from the perspective of the chasing fly - as long as it just follows its moving target and as long as the target does not collide with an obstacle. In other words, if the target - in normal behavior the leading fly - moves on an unobstructed track, the chasing fly can follow the target without jeopardizing its safety as long as it stays on the target's track. Indeed, the flight trajectories of the target fly and the pursuing fly appear to be quite similar, although the similarities have not yet been quantified (Wagner, 1986b; Boeddeker and Egelhaaf, 2005).

\section{ACKNOWLEDGMENTS}

We are grateful to Dr. Norbert Boeddeker and Dr. Jens P. Lindemann for critically reviewing the paper and to the Deutsche Forschungsgemeinschaft (DFG) for financial support.

the Stabilization of Gaze, eds F. A. Miles and J. Wallman (Amsterdam/London/ New York: Elsevier), 239-264.

Collewijn, H., and Tamminga, E. P. (1984) Human smooth and saccadic eyemovements during voluntary pursuit of different target motions on different backgrounds. J. Physiology. 351, 217-250.

Crapse, T. B., and Sommer, M. A. (2008) Corollary discharge across the animal kingdom. Nat. Rev. Neurosci. 9, 587-600.

De Brouwer, S., Missal, M., and Lefevre, P. (2001). Role of retinal slip in the prediction of target motion during smooth and saccadic pursuit. $J$. Neurophysiol. 86, 550-558.

De Brouwer, S., Yuksel, D., Blohm, G., Missal, M., and Lefevre, P. (2002).
What triggers catch-up saccades during visual tracking? J. Neurophysiol. 87, 1646-1650.

Duistermars, B. J., Reiser, M. B.,Zhu, Y., and Frye,M.A. (2007). Dynamic properties of large-field and small-field optomotor flight responses in Drosophila. J. Comp. Physiol. A 193, 787-799.

Egelhaaf, M. (1987). Dynamic properties of two control systems underlying visually guided turning in house-flies. J. Comp. Physiol. A 161, 777-783.

Egelhaaf, M. (1989). Visual afferences to flight steering muscles controlling optomotor response of the fly. J. Comp. Physiol. A 165, 719-730.

Egelhaaf, M. (2009). Insect motion vision. Scholarpedia 4, 1671.

Egelhaaf, M., Hausen, K., Reichardt, W., and Wehrhahn, C. (1988). Visual 
course control in flies relies on neuronal computation of object and background motion. Trends Neurosci. 11, 351-358.

Frye, M. A., and Olberg, R. M. (1995). Visual receptive-field properties of feature detecting neurons in the dragonfly. J. Comp. Physiol. A 177, 569-576.

Gardner, J. L., and Lisberger, S. G. (2001). Linked target selection for saccadic and smooth pursuit eye movements. J. Neurosci. 21, 2075-2084.

Gilbert, C., and Strausfeld, N. J. (1991). The functional organization of malespecific visual neurons in flies. J. Comp. Physiol. A 169, 395-411.

Götz, K. G. (1968). Flight control in Drosophila by visual perception of motion. Kybernetik 4, 199-208.

Gries, M., and Koeniger, N. (1996). Straight forward to the queen: pursuing honeybee drones (Apis mellifera $\mathrm{L}$ ) adjust their body axis to the direction of the queen. J. Comp. Physiol. A 179, 539-544.

Hardie, R. C. (1985). "Functional organization of the fly retina," in Progress in Sensory Physiology, Vol. 5, eds H. Autrum, D. Ottoson, E. R. Perl, R. F. Schmidt, H. Shimazu and W. D. Willis (Berlin/Heidelberg/New York/Tokyo: Springer), 1-79.

Hausen, K. (1982a). Motion sensitive interneurons in the optomotor system of the fly. I. The horizontal cells: structure and signals. Biol. Cybern. 45, 143-156.

Hausen, K. (1982b). Motion sensitive interneurons in the optomotor system of the fly. II. The horizontal cells: receptive field organization and response characteristics. Biol. Cybern. $46,67-79$.

Hausen, K., and Egelhaaf, M. (1989). "Neural mechanisms of visual course control in insects," in Facets of Vision, eds D. Stavenga and R. C. Hardie (Berlin/Heidelberg/New York: Springer), 391-424.

Hausen, K., and Strausfeld, N. J. (1980). Sexually dimorphic interneuron arrangements in the fly visual system. Proc. R. Soc. Lond., B, Biol. Sci. 208, 57-71.

Heisenberg, M., and Wolf, R. (1984). Vision in Drosophila. Berlin/Heidelberg/New York/Tokyo: Springer.

Hengstenberg, R. (1993). "Multisensory control in insect oculomotor systems," in Visual Motion and its Role in the Stabilization of Gaze, eds F.A. Miles and J. Wallman (Amsterdam/Tokyo/New York/London: Elsevier), 285-298.

Ilg, U. J. (1997). Slow eye movement. Prog. Neurobiol. 53, 293-329.

Karmeier, K., van Hateren, J. H., Kern, R., and Egelhaaf, M. (2006). Encoding of naturalistic optic flow by a population of blowfly motion sensitive neurons. J. Neurophysiol. 96, 1602-1614.

Keller, E. L., and Khan, N. S. (1986). Smooth-pursuit initiation in the presence of a textured background in monkey. Vision Res. 26, 943-955.

Kern, R., and Egelhaaf, M. (2000). Optomotor course control in flies with largely asymmetric visual input. J. Comp. Physiol. A 186, 45-55.

Kern, R., van Hateren, J. H., and Egelhaaf, M. (2006). Representation of behaviourally relevant information by blowfly motion-sensitive visual interneurons requires precise compensatory head movements. J. Exp. Biol. 209, 1251-1260.

Kern, R., van Hateren, J. H., Michaelis, C., Lindemann, J. P., and Egelhaaf, M. (2005). Function of a fly motionsensitive neuron matches eye movements during free flight. PLoS Biol. 3, 1130-1138. doi:10.1371/journal. pbio.0030171.

Kimmerle, B., Srinivasan, M. V., and Egelhaaf, M. (1996). Object detection by relative motion in freely flying flies. Naturwissenschaften 83, 380-381.

Kimmerle, B., Warzecha, A.-K., and Egelhaaf, M. (1997). Object detection in the fly during simulated translatory flight. J. Comp. Physiol. A 181, 247-255.

Kimmig, H., Miles, F. A., and Schwarz, U. (1992). Effect of stationary textured backgrounds on the initiation of smooth pursuit eye-movements in monkey. J. Neurosci. 68, 2147-2164.

Kirschfeld, K. (1997). “Course control and tracking: orientation through image stabilization," in Orientation and Communication in Arthropods, ed. M. Lehrer (Basel: Birkhäuser), 67-93.

Kral, K. (2003). Behavioural-analytical studies of the role of head movements in depth perception in insects, birds and mammals. Behav. Processes 64, 1-12.

Krauzlis, R. J. (2004). Recasting the smooth pursuit eye movement system. J. Neurophysiol. 91, 591-603.

Land, M. F. (1993a). Chasing and pursuit in the dolichopodid fly Poecilobothrus nobilitatus. J. Comp. Physiol. A 173 , 605-613.

Land, M. F. (1993b). The visual control of courtship in the fly Poecilobothrus nobilitatus. J. Comp. Physiol. A 173 , 595-603.

Land, M. F. (1999). Motion and vision: why animals move their eyes. J. Comp. Physiol. A 185, 341-352.

Land, M. F., and Collett, T. S. (1974). Chasing behaviour of houseflies (Fannia canicularis). A description and analysis. J. Comp. Physiol. 89 331-357.
Land, M. F., and Collett, T. S. (1997). “A survey of active vision in invertebrates," in From Living Eyes to Seeing Machines, eds M.V. Srinivasan and S. Venkatesh (Oxford: Oxford University Press), 16-36.

Land, M. F., and Eckert, H. (1985). Maps of the acute zones of fly eyes. J. Comp. Physiol. A 156, 525-538.

Land, M. F., and McLeod, P. (2000). From eye movements to actions: how batsmen hit the ball. Nat. Neurosci. 3 1340-1345.

Lindemann, J. P. (2006). Visual Navigation of a Virtual Blowfly. Doctoral thesis, Bielefeld University. URL: http://bieson.ub.uni-bielefeld. de/volltexte/2006/897/

Lindemann, J. P., Kern, R., van Hateren, J. H., Ritter, H., and Egelhaaf, M. (2005). On the computations analysing natural optic flow: quantitative model analysis of the blowfly motion vision pathway. J. Neurosci. 25, 6435-6448.

Lindemann, J. P., Weiss, H., Möller, R., and Egelhaaf, M. (2007). Saccadic flight strategy facilitates collision avoidance: closed-loop performance of a cyberfly. Biol. Cybern. 98, 213-227.

Masson, G., Proteau, L., and Mestre, D. R. (1995). Effects of stationary and moving textured backgrounds on the visuo-oculo-manual tracking in humans. Vision Res. 35, 837-852.

McBeath, M. K., Shaffer, D. M., and Kaiser M.K.(1995). How baseball outfielders determine where to run to catch fly balls. Science 268, 569-573.

Miles, F. A. (1997). Visual stabilization of the eyes in primates. Curr. Opin. Neurobiol. 7, 867-871.

Miles, F. A., and Wallman, J. E. (1993) Visual Motion and its Role in the Stabilization of Gaze. Amsterdam/ London/New York: Elsevier.

Mohrmann, H., and Thier, P. (1995) The influence of structured visual backgrounds on smooth-pursuit initiation, steady-state pursuit and smooth-pursuit termination. Biol. Cybern. 73, 83-93.

Mronz, M., and Lehmann, F. O. (2008) The free-flight response of Drosophila to motion of the visual environment. J. Exp. Biol. 211, 2026-2045.

Niemann, T., and Hoffmann, K.-P (1997). Motion processing for saccadic eye movements during the visually induced sensation of egomotion in humans. Vision Res. 37 3163-3170.

Nordström, K., Barnett, P. D., and O'Carroll, D. C. (2006). Insect detection of small targets moving in visual clutter. PLoS Biol. 4, 378-386. doi:10.1371/journal.pbio.0040054.

Nordström, K., and O'Carroll, D. C. (2006).Small object detection neurons in female hoverflies. Proc. R. Soc. Lond B, Biol. Sci. 273, 1211-1216.

O'Carroll D. C. (1993). Feature-detecting neurons in dragonflies. Nature 362, 541-543.

Olberg, R. M. (1981). Object- and selfmovement detectors in the ventral nerve cord of the dragonfly. J. Comp. Physiol. 141, 327-334.

Olberg, R. M., Worthington, A. H., Fox, J. L., Bessette, C. E., and Loosemore, M. P. (2005). Prey size selection and distance estimation in foraging adult dragonflies. J. Comp. Physiol. A 191, 791-797.

Olberg, R. M., Worthington, A. H., and Venator, K.R. (2000). Prey pursuit and interception in dragonflies. J. Comp. Physiol. A 186, 155-162.

Orban de Xivry, J.-J., and Lefèvre, P. (2007). Saccades and pursuit: two outcomes of a single sensorimotor process. J. Physiol. 584, 11-23.

Reichardt, W., Poggio, T., and Hausen, K. (1983). Figure-ground discrimination by relative movement in the visual system of the fly. Part II: Towards the neural circuitry. Biol. Cybern. 46(Suppl.), $1-30$.

Rossel, S. (1980). Foveal fixation and tracking in praying mantis. J. Comp. Physiol. 139, 307-331.

Schilstra, C., and van Hateren, J.H. (1999) Blowfly flight and optic flow. I. Thorax kinematics and flight dynamics. J. Exp. Biol. 202, 1481-1490.

Schweigart, G., Mergner, T., Evdokimidis, I., Morand, S., and Becker, W. (1997) Gaze stabilization by optokinetic reflex (OKR) and vestibulo-ocular reflex (VOR) during active head rotation in man. Vision Res. 37, 1643-1652.

Shaffer, D. M., and McBeath, M. K. (2002). Baseball outfielders maintain a linear optical trajectory when tracking uncatchable fly balls. J. Exp. Psychol. Hum. Percept. Perform. 28, 335-348.

Sherman,A., and Dickinson, M.H. (2003). A comparison of visual and halteremediated equilibrium reflexes in the fruit fly Drosophila melanogaster. J. Exp. Biol. 206, 295-302.

Spering, M., and Gegenfurtner, K. R. (2007). Contrast and assimilation in motion perception and smooth pursuit eye movements. J. Neurophysiol. 98, 1355-1363.

Srinivasan, M. V., Lehrer, M., Kirchner, W. H., and Zhang, S. W. (1991). Range perception through apparent image speed in freely flying honeybees. Vis. Neurosci. 6, 519-535.

Stout, J., Atkins, G., Weber, T., and Huber, F. (1987). The effect of visual input on calling song attractiveness for female Acheta domesticus. Physiol. Entomol. 12, 135-140. 
Strausfeld, N. J. (1991). Structural organization of male-specific visual neurons in calliphorid optic lobes. J. Comp. Physiol. A 169, 379-393.

Strauss, R., and Heisenberg, M. (1990). "Gaze stabilizing head movements compensate for walk-induced body oscillations in the fly Drosophila melanogaster," in Brain-PerceptionCognition, eds N. Elsner and G. Roth (Stuttgart/New York: Thieme), 63.

Tammero, L. F., and Dickinson, M. H. (2002). The influence of visual landscape on the free flight behavior of the fruit fly Drosophila melanogaster. J. Exp. Biol. 205, 327-343.

Trischler, C. (2009). Chasing Control in Male Blowflies: Behavioural Performance and Neuronal Responses. Doctoral dissertation, Bielefeld University. URL: http://bieson.ub.unibielefeld.de/volltexte/2009/1432/

Trischler, C., Boeddeker, N., and Egelhaaf, M. (2007). Characterisation of a blowfly male-specific neuron using behaviourally generated visual stimuli. $J$. Comp. Physiol. A 193, 559-572.

van Hateren, J.H., and Schilstra, C. (1999). Blowfly flight and optic flow. II. Head movements during flight. J. Exp. Biol. 202, 1491-1500.
Virsik, R., and Reichardt, W. (1976). Detection and tracking of moving objects by the fly Musca domestica. Biol. Cybern. 23, 83-98.

von Holst, E., and Mittelstaedt, $\mathrm{H}$. (1950). Das Reafferenzprinzip (Wechselwirkungen zwischen Zentralnervensystem und Peripherie). Naturwissenschaften 37, 464-476.

Wachenfeld, A., and Hausen, K. (1993). "Functional characterization of the male lobula giant neuron 1 (MLG1) in the blowfly, Calliphora erythrocephala (Meig.)," in Göttingen Neurobiology Report, eds N.Elsner and M.Heisenberg (Stuttgart/New York: Thieme), 359.

Wachenfeld, A., and Hausen, K. (1994). "The role of male-specific visual interneurons in the mating behavior of the blowfly, Calliphora erythrocephala (Meig.)," in Göttingen Neurobiology Report, eds N. Elsner and H. Breer (Stuttgart/New York: Thieme), 440.

Wagner, H. (1986a). Flight performance and visual control of flight of the freeflying housefly (Musca domestica). I. Organization of the flight motor. Philos. Trans. R. Soc. Lond., B, Biol. Sci. 312, 527-551.

Wagner, H. (1986b). Flight performance and visual control of flight of the free-flying housefly (Musca domestica) II. Pursuit of targets. Philos. Trans. $R$. Soc. Lond., B, Biol. Sci. 312, 553-579.

Wagner, H. (1986c). Flight performance and visual control of flight of the freeflying housefly (Musca domestica). III. Interactions between angular movement induced by wide- and smallfield stimuli. Philos. Trans. R. Soc. Lond., B, Biol. Sci. 312, 581-595.

Warzecha,A.-K., and Egelhaaf,M. (1996). Intrinsic properties of biological motion detectors prevent the optomotor control system from getting unstable. Philos. Trans. R. Soc. Lond., B, Biol. Sci. 351, 1579-1591.

Webb, B., Harrison, R. R., and Willis, M.A. (2004). Sensorimotor control of navigation in arthropod artificial systems. Arthropod Struct. Dev. 33, 301-329.

Weber, T., Atkins, G., Stout, J., and Huber, F. (1987). Female Acheta domesticus track acoustical and visual targets with different walking modes. Physiol. Entomol. 12, 141-147.

Wehner, R. (1981). “Spatial vision in arthropods," in Handbook of Sensory Physiology, Vol.VII/6C, Comparative Physiology and Evolution of Vision in Invertebrates, ed. H. Autrum (Berlin/Heidelberg/New York: Springer), 287-616.
Zeil, J. (1983). Sexual dimorphism in the visual system of flies: the free flight behaviour of male Bibionidae (Diptera). J. Comp. Physiol. A 150, 395-412.

Conflict of Interest Statement: The authors declare that the research was conducted in the absence of any commercial or financial relationships that could be construed as a potential conflict of interest.

Received: 15 November 2009; paper pending published: 18 January 2010; accepted: 09 April 2010; published online: 14 May 2010.

Citation: Trischler $C$, Kern $R$ and Egelhaaf M (2010) Chasing behaviour and optomotor following in free-flying male blowflies: flight performance and interactions of the underlying control systems. Front. Behav. Neurosci. 4:20. doi: 10.3389/fnbeh.2010.00020

Copyright (c) 2010 Trischler, Kern and Egelhaaf. This is an open-access article subject to an exclusive license agreement between the authors and the Frontiers Research Foundation, which permits unrestricted use, distribution, and reproduction in any medium, provided the original authors and source are credited. 